\title{
Sinopsis de los principios pedagógicos aplicados en la praxis
}

\section{Synopsis of pedagogical principles applied in praxis}

\author{
Gianina Lorena Carpio Guacon ${ }^{1}$ \\ gianina lorena24@hotmail.com \\ Omar Reinaldo Desiderio Triviño ${ }^{2}$ \\ omar 0019@hotmail.com \\ Sony Steeven Rack Cottallat 3 \\ sonyrack1993@hotmail.com
}

Recibido: 1/04/219; Aceptado: 1/06/2019

\begin{abstract}
RESUMEN
Estudiar pedagogía es más allá de pizarras, marcadores y libretas. Ser docente es marcar un precedente; ser ese factor de cambio desde las aulas de clases a la sociedad con un aporte concreto, real y profesional. El alcance que puede tener un docente con sus estudiantes es lo que hace esta carrera tan importante y especial. Cuando entran a la universidad para estudiar pedagogía se dan cuenta de dos puntos clave: que la educación es un mundo infinito por descubrir y que no solo en el aula de clases sino en el contexto educativo como tal. Los futuros docentes deben tener en claro que al ejercer dicha profesión además de cumplir un sinnúmero de roles, también deben regirse a normas o reglas que la profesión exige. Deben seguirse pasos para la formación de sus educandos. Por ello el presente trabajo abordará un tema importante que son los principios pedagógicos y como lo ejecutan los docentes en el campo práctico.
\end{abstract}

Palabras Clave: pedagogía, profesión, docentes, principios

\begin{abstract} perform it in the practical field.

\footnotetext{
${ }^{1}$ Universidad de Guayaquil, Guayaquil, Ecuador.

2 Universidad de Guayaquil, Guayaquil, Ecuador.

${ }^{3}$ Universidad de Guayaquil, Guayaquil, Ecuador.
}

Studying pedagogy is beyond blackboards, markers and notebooks. Being a teacher is setting a precedent, being a factor of change from classrooms to society with a concrete, real and professional contribution. The scope that a teacher can have with his students is what makes this career so important and special. When he enters the university to study pedagogy they realize two key points: education is an infinite world to discover and that not only in the classroom but in the educational context as such. Future teachers must be clear that in exercising this profession in addition to fulfilling a number of roles, they must also follow the rules or rules that the profession requires, they must follow steps for the training of their students. Therefore, this paper addresses an important issue that is the pedagogical principles and how teachers 


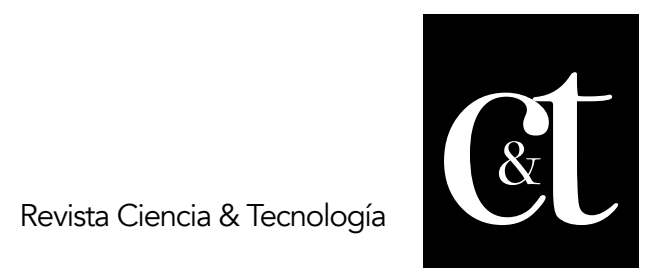

No. 23, 31 de julio de 2019

ISSN impreso: 1390 - 6321

ISSN online: 2661 - 6734

Keywords: Pedagogy, profession, teachers, principles

\section{Introducción}

La pedagogía es una disciplina que reconoce en las cosas de todos los días problemas profundos y complejos. Hacer pedagogía es reflexionar sobre la educación, así como se dice que la filosofía es la madre de todas las ciencias, se puede decir que la pedagogía es la madre de todas las ciencias y disciplinas de la educación (Calzadilla, 2004).

Estudiar pedagogía es más allá de pizarras, marcadores y libretas, ser docente es marcar un precedente, ser ese factor de cambio desde las aulas de clases a la sociedad con un aporte concreto, real y profesional. El alcance que puede tener un docente con sus estudiantes es lo que hace esta carrera tan importante y especial, cuando entran a la universidad para estudiar pedagogía se dan cuenta de dos puntos clave, que la educación es un mundo infinito por descubrir y no solo en el aula de clases si no en el contexto educativo como tal.

Además, de ello el campo práctico es más difícil de lo que la teoría hace ver, puesto que el docente cree llevar todas las preguntas listas para su clase y al momento de impartirla se encuentra con un sin número de personalidades en el salón es ahí cuando se da cuenta que él no está para hacer las preguntas sino para responderlas.

Los futuros docentes deben tener en claro que al ejercer dicha profesión además de cumplir un sin número de roles, también deben regirse a normas o reglas que la profesión exige, deben seguirse pasos para la formación de sus educandos. Por ello el presente trabajo abordara un tema importante que son los principios pedagógicos y como lo ejecutan los docentes en el campo práctico.

Los principios pedagógicos son condiciones esenciales para la implementación del currículo, son reglas o normas que ayudan en la transformación de la práctica docente, el logro de los aprendizajes y mejorar la práctica educativa. En este sentido los principios actúan como guía de las metas que el hombre debe lograr a través de su actividad, para la transformación y creación del nuevo proceso a través el cual el hombre no solo transforma el medio sino se auto transforma. (Celeiro, 2019).

Para poder observar la ejecución de estos principios pedagógicos los estudiantes pertenecientes a la Universidad de Guayaquil, Facultad de Filosofía, letras y ciencias de la Educación de la Carrera "Pedagogía de las ciencias experimentales de la Química y la Biología" del tercer semestre formularon una guía de observación la cual consta con indicadores referentes a cada principio pedagógico, de los cuales se abordaran tres en el presente trabajo esta acción se pudo realizar mediante la práctica pre-profesional en la institución educativa "Academia Naval Almirante Illingworth".

\section{Desarrollo}

Para los docentes al momento de impartir una clase es primordial mantener a todos sus estudiantes con el interés de aprender $y$, o reforzar conocimientos ya planteados para ello surgen una serie de dudas ¿cómo hacerlo?; ¿Para qué hacerlo?; ¿Es posible?.

La principal tarea que tiene el docente es mantener el interés en el educando durante el periodo lectivo, evitando distracción para la correcta aplicación de los objetivos. Muchas experiencias a lo largo de esta profesión plantean que es difícil mantener al 


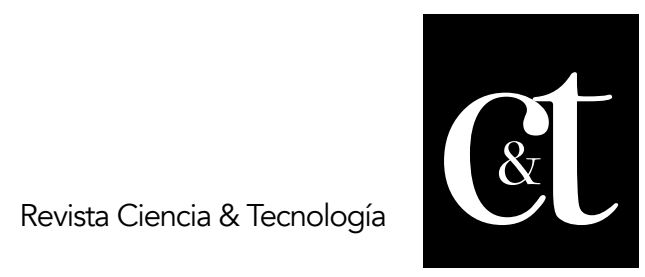

No. 23, 31 de julio de 2019

ISSN impreso: 1390 - 6321

ISSN online: 2661 - 6734

estudiante totalmente activo pero no es imposible, para la ejecución de esta práctica se necesita tino y empatía con los educandos además del respeto que existan entre ambos.

De este antecedente surge uno de los indicadores más relevantes en la guía de observación aplicada "El docente mantiene el interés del estudiante" a través de la indagación, se pudo constatar que él docente mientras impartía su asignatura empoderaba de ella, como mediante la motivación lograba captar el interés de sus estudiantes a pesar de que había una cantidad de estudiante considerable en todo momento se mantuvo el interés hacia la clase, se notaba esa empatía con el docente y la participación de los educandos, pero a pesar de que existía una gran simpatía y entusiasmo en el salón de clases era evidente que otro aspecto importante se pudo observar: "respeto y orden".

El docente además de ser una guía en el proceso de aprendizaje para los educandos, es un modelo a seguir, esa persona que directa o indirectamente refuerza o crea hábitos, valores y principios. Para establecer una correcta y sana convivencia el docente debe dejar en claro cuáles serán las reglas y normas que se mantendrán durante el periodo electivo, pero como se mencionó anteriormente para una correcta ejecución de estas reglas es necesario que el docente lo practique con el ejemplo (Barreda, 2012).

Sobre lo expuesto anteriormente se pudo observar en la praxis la correcta ejecución de este indicador, se observó un ambiente de respeto y entendimiento mutuo, no solamente en el tema de la asignatura, sino en general, se notaba los buenos principios desde la forma en cómo se expresaban, hasta la puntualidad de ambos sujetos.

El ambiente que se pudo observar en aquella clase llevó a verificar la ejecución de un indicador que suele ser clave para la evaluación que realice el docente, el desarrollo individual y grupal de sus estudiantes, donde además de evaluarlos por sus conocimientos y trabajos teóricos-prácticos se puede evaluar su compañerismo, respeto y honestidad que existe entre los educandos. Los trabajos en equipo dentro del aula fomentan habilidades como la comunicación y la resolución de conflictos, pero también sirven para desarrollar conceptos teóricos. Pero no todo trabajo en grupo funciona como camino para un mayor y mejor aprendizaje. La clave, señalan los expertos, es que se genere una interdependencia entre los miembros del grupo: que todos dependan del trabajo de todos (Blas-López, 2012). Esta es la base sobre la que se construye el concepto del aprendizaje cooperativo, una metodología en auge que defiende los beneficios de aprender en equipo frente a los tradicionales enfoques individualistas o competitivos. Es un sistema muy concreto de trabajo en grupo en el que los miembros dependen unos de otros. No se trata de que cada uno haga su tarea de forma independiente y luego la junten, sino de que se genere una interdependencia, señala Sánchez (2018). Mediante aquello es necesario dejar en claro que, así como se evalúa el trabajo grupal también lo hacen en la parte individual en su gran mayoría con trabajos autónomos, ya que en la actualidad se busca que las personas interactúen no solo en el área educativa sino en todo aspecto de sus vidas pero que mejor manera que fortalecer esa interacción en las aulas de clase. Este antecedente con el cual se pudo plantear el indicador expuesto, se observó que los estudiantes de bachillerato trabajan de forma 


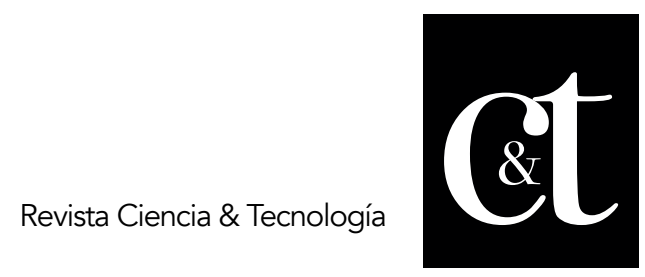

No. 23, 31 de julio de 2019

ISSN impreso: 1390 - 6321

ISSN online: 2661 - 6734

Cooperativa, incluso cuando es momento de hacerlo de manera individual ellos motivaban a sus compañeros para avanzar todos juntos como curso, el sentido de liderazgo los caracterizo en la asignatura impartida.

La conexión de lo que se aprende con su lado práctico es muy importante para captar la atención del alumno. Muchas veces, el problema de la falta de compromiso con la clase tiene que ver con que el alumno no ve la aplicación práctica o la importancia que tiene lo impartido para su desarrollo. Esto los lleva a querer aprender de una manera fácil, memorizar la información y estudiar o salvar haciendo lo mínimo posible. Por lo tanto, despertar en el estudiante el sentimiento de compromiso con el aprendizaje será tarea del docente.

Los profesores serán los encargados de cautivar la atención y despertar la curiosidad del aprendizaje en los estudiantes, el educador tiene que vincular su mensaje educativo con la vida, pues de lo contrario, este le llegara vacío, abstracto, carente de significación para él y por lo tanto no se implicara en la tarea de aprendizaje; deberá aprovechar el factor más importante que influye en el aprendizaje, es lo que el alumno ya sabe. Determinar esto y enseñarle en consecuencia, según (Ausubel, 2008). Ausubel consideraba que el aprendizaje de nuevos conocimientos se basa en lo que ya es conocido con anterioridad. Es decir, la construcción del conocimiento comienza con la observación, registro de acontecimientos y objetos a través de conceptos que ya tenemos. Aprender mediante la construcción de una red de conceptos y añadiendo nuevos a los existentes.

En este indicador donde el docente relaciona la clase con la vida cotidiana se pudo observar que en la institución que de manera general no solo un docente aplica este tipo de enseñanza sino varios y es algo positivo para los estudiantes. Es más como se lo mencionó en indicadores anteriores esto ayuda a mantener el interés en los educandos. Es así como todos los principios pedagógicos están relacionados para mejorar la práctica educativa.

De acuerdo con la pedagogía activa, se revela en el aula la importancia que tiene que los estudiantes sean participativos, activos protagonistas de su aprendizaje, "No basta que las personas aprendan se requieren que aprendan a aprender" (Red educacional Ignaciana, 2010, pág. 34). Hay que invitar a los estudiantes a participar en clase, realizar comentarios verbales e involucrarse en la conversación para que no haya solo una exposición por parte del docente. Hay que promover la comunicación entre profesores y estudiantes. Una manera de hacerlo es a través del planteo de dudas, preguntas que no hayan realizado en clase y comentarios a través del correo electrónico o plataformas educativas, por ejemplo. Será importante recordarles a los alumnos que escuchar es también parte de la participación y que es necesario desarrollar esa capacidad.

Mientras se aplicó el instrumento se observó que una gran mayoría de estudiantes se notaban participativos y atentos a la asignatura, hacían preguntas acerca del tema y respondían con entusiasmo.

Las estrategias de enseñanza se definen como los procedimientos o recursos utilizados por los docentes para lograr aprendizajes significativos en los alumnos. Cabe hacer mención que el empleo de diversas estrategias de enseñanza permite a los docentes lograr un proceso de aprendizaje activo, participativo, de cooperación y 


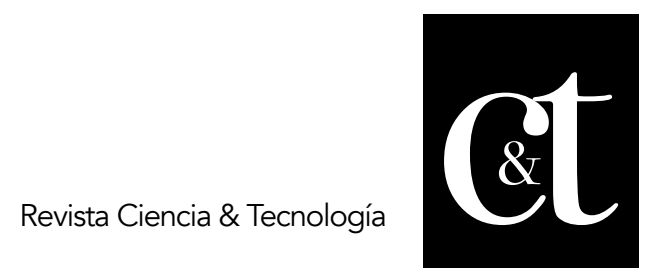

No. 23, 31 de julio de 2019

ISSN impreso: 1390 - 6321

ISSN online: 2661 - 6734

vivencial. Las vivencias reiteradas de trabajo en equipo cooperativo hacen posible el aprendizaje de valores y afectos que de otro modo es imposible de lograr.

Es importante destacar que las estrategias como recurso de mediación deben de emplearse con determinada intensión, y por tanto deben de estar alineadas con los propósitos de aprendizaje, así como con las competencias a desarrollar.

Cabe subrayar la importancia que representa el papel del docente en el proceso enseñanza aprendizaje ya que, en el desarrollo de una sesión de clase, el docente debe crear ambientes de aprendizaje propicios para aprender.

Las estrategias para activar los conocimientos previos en los estudiantes interrogantes son la lluvia de ideas, estos recursos son importantes porque permiten llamar la atención o distraer, las ilustraciones son más recomendadas que las palabras para comunicar ideas de tipo concreto o de bajo nivel de abstracción conceptos de tipo visual o espacial, además promueven el interés y motivación.

Todo ello conlleva a la utilización de estrategias docentes y métodos que propicien un aprendizaje intencional, reflexivo, consciente y autorregulado, regido por objetivos y metas propios, como resultado del vínculo entre lo afectivo y lo cognitivo, de las interacciones sociales y la comunicación, que tengan en cuenta la diversidad del estudiantado y las características de la generación que está en las aulas, con la irrupción de tecnologías de la información y las comunicaciones. Esto les imprime cierta autonomía en la búsqueda de información, lo cual no siempre es aprovechado por el docente $y$, por tanto, el proceso enseñanza-aprendiza no se adecua a la realidad que impone el nuevo siglo.

Desde el punto de vista educativo es necesario entonces, utilizar cada vez más en las aulas, herramientas propias de esta generación, para motivarlos y desarrollar sus habilidades a partir de sus estilos, los modos y formas en que aprenden. En las estrategias docentes se interrelacionan dialécticamente en un plan global los objetivos que se persiguen, los recursos didácticos, métodos de enseñanza-aprendizaje y actividades para alcanzarlos, a partir de fases o etapas relacionadas con las acciones de orientación, ejecución y control de la actividad de aprendizaje.

\section{Conclusiones}

Para concluir con el presente trabajo se expresa que la pedagogía es fundamental en el proceso de acompañamiento del individuo, para que este se entusiasme a querer aprender y desarrolle mejor sus capacidades de aprendizaje. Además, que tiene como objetivo explicar el proceso educativo, por ende, le sirve al educador para saber sus falencias en su método de enseñar y de tal manera mejorar. Gracias a la observación que se realizó en la institución educativa (ANAI) se pudo vivenciar más de cerca lo que sucede en el salón de clases, pese a que hubo muchos aspectos positivos hay que reflexionar acerca de nuevos métodos de enseñanza, lo que se ha transformado actualmente en una tarea más del docente. Entre las distintas herramientas con las que cuenta, desde estas líneas se aboga por la realización de actividades consideradas divertidas o distendidas por los alumnos, las cuales no entorpecen su aprendizaje ni rendimiento, sino todo lo contrario. Los alumnos se involucran en la actividad propuesta porque el tema les resulta interesante y el "envoltorio" atractivo. Son capaces de formular sus propias definiciones o crear sus propias preguntas, con información que ellos mismos han buscado. 


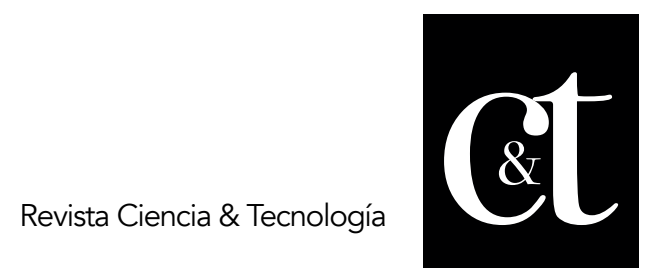

No. 23, 31 de julio de 2019

ISSN impreso: 1390 - 6321

ISSN online: 2661 - 6734

Las habilidades de trabajo grupal, la colaboración de los iguales en el aula, la mayor motivación que le alumno experimenta lal otorgarle mayor autonomía en el aprendizaje de los contenidos específicos en la asignatura, unidas al desempeño en argumentar y desarrollar ideas, exponerlas e incluso evaluando a sus propios compañeros, constituyen otros aspectos relevantes de las actividades propuestas.

\section{Referencias bibliográficas}

Asubel, D. (2002). Adquisición y retención del conocimiento: una perspectiva cognitiva. Madrid; España. Editorial Paidos.

Barreda, M. (2012). El docente como gestor del clima del aula: factores a tener en cuenta (tesis de maestría). Universidad de Cantabria. España.

Blas-López, Y. (2012). Estrategias para trabajar en equipo dentro del aula. Cinzontle. Vol. Julio-diciembre (12-14). Descargado de:

revistas.ujat.mx/index.php/Cinzontle/article/download/2615/2046.

Calzadilla, R. (2004). La pedagogía como ciencia humanista: conocimiento de síntesis, complejidad y pluridisciplinariedad. Revista de Pedagogía. Vol. 25(72), pp. 123-148.

Celeiro, A. (2019). Material de apoyo a la asignatura de Pedagogía.

Sánchez, B. (18 de Julio de 2018). El País. En grupo se aprende más (y mejor).

Recuperado

https://elpais.com/economia/2018/07/17/actualidad/1531835822_840423 